\title{
Normal Mode Analysis of Generalized Magneto-Thermoelastic Medium with Initial Stress Under Green-Naghdi Theory
}

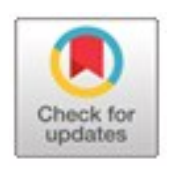

\author{
Alzaerah. Ramadhan Mohammed. Aldeeb.
}

\author{
Department of Mathematics, faculty of education Alzawia University, Libya
}

Received: 16 December 2020 / Accepted: 30 December 2020

Doi: https://doi.org/10.54172/mjsc.v35i4.330

\begin{abstract}
The normal mode analysis method was used to study the effect of both the initial stress and the magnetic field on a thermally elastic body. This method is used to obtain the exact expressions for the considered variables. Some particular cases are also discussed in the context of the problem. The generalized thermal elasticity equations were reviewed under the influence of the basic initial stress and the magnetic field using the theory (Green-Naghdi) of the second and third types (the second type with no energy dispersion and the third type with energy dispersion). The different physical quantities were illustrated in the presence and absence of both the initial stress and the magnetic field. The results of this research show the extent of difference between the second and third types of Green and Naghdi's theory. All results and figures were obtained using (MATLAB R2013a) program.
\end{abstract}

Keywords: Generalized Thermo-Elasticity; Magnetic Field; Initial Stress; Normal Mode Analysis; Green and Naghdi Theory.

\section{INTRODUCTION}

The generalized theory of thermoelasticity is one of the modified versions of the classical uncoupled and coupled theory of thermoelasticity and has been developed in order to remove the paradox of physical impossible phenomena of the infinite velocity of thermal signals in the classical coupled thermoelasticity. (Hetnarski \& Ignaczak, 1999) examined five generalizations of the coupled theory of thermoelasticity. The first generalization was proposed by (Lord \& Shulman, 1967), which involves one relaxation time for a thermoelastic process. The second generalization is due to (Green \& Lindsay, 1972) which takes into account two relaxation times. The third generalization of the coupled theory of thermoelasticity was introduced by (Green \& Naghdi, 1993), who developed different theories labeled type I, type II, and type III. The (G-N I) theory in the linearized theory is equivalent to the classical coupled thermoe- lasticity theory. The (G-N II) theory does not admit energy dissipation, while the third (G$\mathrm{N}$ III) theory admits dissipation of energy. The heat flux is a combination of type I and type II. Both type II and type III theories imply a finite speed of propagation for heat waves. (Bargmann \& Steinmann, 2006) investigated the $(\mathrm{G}-\mathrm{N})$ approach for modeling the phenomenon of second sound.(Othman \& Atwa, 2011; Othman \& Atwa, 2012; Othman et al., 2013b; Othman \& Kumar, 2009), has discussed different problems for various materials with different effects using the $(\mathrm{G}-\mathrm{N})$ theory. The fourth generalization of the coupled theory of thermoelasticity was developed by (Chandrasekharaiah, 1998; Tzou, 1995).

Initial stress in solids has a significant influence on the mechanical response of the material from an initially stressed configuration and has applications in geophysics, engineering structures, and the behavior of soft biological tissues. Initial stress arises from pro-

*Corresponding Author: Alzaerah. Ramadhan . Mohammed. Aldeeb.a.aldeeb@zu.edu.ly_78@yahoo.com, Department of Mathematics, faculty of education Alzawia University, Libya . 
cesses, such as manufacturing or growth, and is present in the absence of applied loads. (Montanaro, 1999) formulated the isotropic thermoelasticity with hydrostatic initial stress. (Ailawalia et al., 2009; Othman \& Song, 2007; Singh, 2008; Singh et al., 2006), and many others have applied (Montanaro, 1999) theory to study the plane harmonic waves in the context of generalized thermoelasticity. (Othman \& Atwa, 2012) investigate the effect of initial stress under the GreenNaghdi (G-N) theory for different cases in thermoelasticity. (Ailawalia \& Narah, 2009) studied the effect of hydrostatic initial stress and rotation in a generalized thermoelastic medium. (Othman \& Edeeb, 2016) studied the Effect of Initial Stress on Generalized Magneto-thermoelasticity Medium with Voids: A Comparison of Different Theories. (AbdElaziz et al., 2019) studied the On the Effect of Thomson and Initial Stress in a ThermoPorous Elastic Solid under G-N Electromagnetic Theory.

The theory of magneto-thermoelasticity is concerned with the interacting effects of the applied magnetic field on the elastic and thermoelastic deformations of a solid body. This theory has aroused much interest in many industrial appliances, particularly in nuclear devices where there exists a primary magnetic field; various investigations are to be carried out by considering the interaction between magnetic, thermal, and strain fields. Analyses of such problems also influence various applications in biomedical engineering as well as in different geomagnetic studies. The development of the interaction of electromagnetic field, the thermal field, and the elastic field is available in many works such as (Abd-Alla et al., 2003; Choudhuri \& Debnath, 1985; Othman \& Song, 2006; Paria, 1966; Sherief \& Helmy, 2002) studied the effect of rotation on the reflection of magneto-thermoelastic waves under thermoelasticity without energy dissipation with the (G-N) theory of type II. (Othman \& Kumar, 2009) studied the reflection of magneto- thermoelastic waves with temperaturedependent properties in the context of generalized thermoelasticity with (G-N) theory of type II, i.e. without energy dissipation, and other models of thermoelasticity. (Othman \& Atwa, 2011) studied the effect of the magnetic field on the two-dimensional problem of generalized thermoelasticity without energy dissipation. (Othman et al., 2013a) studied the generalized magneto-thermo-microstretch elastic solid under a gravitational field with energy dissipation. Recently (Othman et al., $2013 \mathrm{~b}$ ) studied the effect of magnetic field and rotation on generalized thermomicrostretch elastic solid for a mode-I crack using (G-N) theory. (Atwa, 2014) studied the generalized magneto-thermoelasticity with two temperatures and initial stress under GreenNaghdi theory. (Abo-Dahab et al., 2017) studied A Two-Dimensional Problem with Rotation and Magnetic Field in the Context of Four Thermoelastic Theories, the normal-mode analysis method was applied to obtain the exact solutions for the physical problem.

\section{FORMULATION OF THE PROBLEM AND BASIC EQUATIONS}

Consider an isotropic, homogeneous, linear, thermally, and electrically conducting thermoelastic half-space $(x \geq 0,-\infty \leq y \leq \infty)$. The rectangular Cartesian coordinate system $(x, y, z)$, having originated on the surface $z=0$, for the two dimensional problem assume the dynamic displacement vector as $u=(u, v, 0)$. The surface $(x=0)$ of the half-space is taken to be traction-free and subjected to mechanical and thermal loads. All the considered functions are assumed to be bounded as $x \rightarrow \infty$. The whole body is at a constant temperature $T_{0}$. Consider also that the orientation of the primary magnetic field $\boldsymbol{H}=\left(0,0, H_{0}\right)$ is towards the positive direction of $z$-axis. Due to the application of this magnetic field, an induced magnetic field $\boldsymbol{h}$ and an induced electric field $\boldsymbol{E}$. arise in the medium .All the considered functions will 
depend on time ${ }^{t}$ and the coordinates $x$ and $y$. So the displacement vector $\boldsymbol{u}$ has the components

$u_{x}=u(x, z, t), \quad u_{y}=v(x, z, t), u_{z}=0$.

The variation of the magnetic and electric fields are a perfectly conducting slowly moving medium and are given by Maxwell's equations:

$\operatorname{curl} \boldsymbol{h}=\boldsymbol{J}+\varepsilon_{0} \dot{\boldsymbol{E}}$,

$\operatorname{curl} \boldsymbol{E}=-\mu_{0} \dot{\boldsymbol{h}}$,

$\boldsymbol{E}=-\mu_{0}(\dot{\boldsymbol{u}} \boldsymbol{H}$

$\operatorname{div} \boldsymbol{h}=0$.

From the above equations, one can obtain

$$
\begin{aligned}
& \boldsymbol{E}=\mu_{0} H_{0}\left(v, 0,-\imath^{-}\right. \\
& \boldsymbol{h}=\left(0,0,-H_{0} e\right),
\end{aligned}
$$

$$
\boldsymbol{J}=\left(-h_{, \mathrm{y}}-\varepsilon_{0} \mu_{0} H_{0} \ddot{v}, 0, h_{, \mathrm{x}}+\varepsilon_{0} \mu_{0} H_{0} \ddot{\imath}\right.
$$

The constitutive relations are given by

$$
\begin{aligned}
& \sigma_{i j}=2 \mu e_{i j}+\delta_{i j}\left[\lambda e_{k k}-\beta \frac{\partial T}{\partial x}\right]-P\left(\delta_{i j}+\omega_{i j}\right) \\
& e_{i j}=\frac{1}{2}\left(u_{i, j}+u_{j, i}\right), \quad \omega_{i j}=\frac{1}{2}\left(u_{j, i}-u_{i, j}\right),
\end{aligned}
$$

The equation of motion has the form

$$
\sigma_{j i, j}+F_{i}=\rho^{\prime \prime} . \quad i, j=1,2,3 \text {. }
$$

Where $F_{i}$ is the Lorentz force and is given by: $\quad F_{i}=\mu_{0}(\boldsymbol{J} \times \boldsymbol{H})_{\mathrm{i}}$.

From equations (8) and (11), Lorentz force is obtained

$$
\begin{aligned}
& \boldsymbol{F}=\left(F_{x}, F_{y}, F_{z}\right)=\left(\mu_{0} H_{0}^{2} e_{, \mathrm{x}}-\right. \\
& \left.\varepsilon_{0} \mu_{0}^{2} H_{0}^{2}{ }^{\imath} \quad{ }_{0}^{2} e_{, \mathrm{y}}-\varepsilon_{0} \mu_{0}^{2} H_{0}^{2} \ddot{v}, 0\right) .
\end{aligned}
$$

Substituting from equations (9) and (13) into equation (11), the equations of motion can be written as follows

$$
\begin{gathered}
\left(\mu-\frac{P}{2}\right) \nabla^{2} u+\left(\lambda+\mu+\mu_{0} H_{0}^{2}+\frac{P}{2}\right) e_{, x} \\
-\beta T_{, x}=\rho\left(1+\frac{\varepsilon_{0} \mu_{0}^{2} H_{0}^{2}}{\rho}\right) i{ }^{*}
\end{gathered}
$$

$$
\begin{gathered}
\left(\mu-\frac{P}{2}\right) \nabla^{2} v+\left(\lambda+\mu+\mu_{0} H_{0}^{2}+\frac{P}{2}\right) e_{, y} \\
-\beta T_{, y}=\rho\left(1+\frac{\varepsilon_{0} \mu_{0}^{2} H_{0}^{2}}{\rho}\right) \ddot{v} .
\end{gathered}
$$

The equation of heat conduction has the form

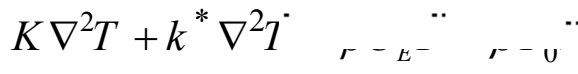

Where, $\sigma_{i j}$ are the stress tensor components, $e_{i j}$ are the strain tensor components, $\omega_{i j}$ is the rotation tensor, $e=e_{k k}$ is the cubic dilatation, $\delta_{i j}$ is Kronecker's delta, $u_{i}$ is the displacement vector, $\lambda, \mu$ are the elastic constants, $T$ is the absolute temperature, $T_{0}$ is the temperature of medium in its natural state assumed to be such that $\left|\left(T-T_{0}\right) / T_{0}\right|<1$,

$\varepsilon_{0}, \mu_{0}$ are the electric and magnetic permeability respectively, $\boldsymbol{J}$ is the current density vector, $\boldsymbol{E}$ is the induced electric field vector, $\boldsymbol{h}$ is the induced magnetic field vector, $H_{0}$ is a constant magnetic field, $P$ is the initial stress, $\lambda, \mu$ are Lame's constants, $\beta=(3 \lambda+2 \mu) \alpha_{t}, \alpha_{t}$ is the coefficient of linear thermal expansion, $\rho$ is the density, $C_{E}$ is the specific heat at constant strain, $k$ is the coefficient of thermal conductivity, $k^{*}$ is the material constant characteristic of the theory, and $\nabla^{2}=\frac{\partial^{2}}{\partial x^{2}}+\frac{\partial^{2}}{\partial y^{2}}+\frac{\partial^{2}}{\partial z^{2}}$.

When $k^{*} \rightarrow 0$ then equation (16) reduces to the heat conduction equation in $(\mathrm{G}-\mathrm{N})$ theory (of type II),

$\nabla=\frac{\partial}{\partial x} i+\frac{\partial}{\partial y} j$. and $\nabla^{2}=\frac{\partial^{2}}{\partial x^{2}}+\frac{\partial^{2}}{\partial y^{2}}$.

The components of stress tensor are

$$
\begin{aligned}
& \sigma_{x x}=\lambda\left(\frac{\partial u}{\partial x}+\frac{\partial v}{\partial y}\right)+2 \mu \frac{\partial u}{\partial x}-\beta T-p, \\
& \sigma_{y y}=\lambda\left(\frac{\partial u}{\partial x}+\frac{\partial v}{\partial y}\right)+2 \mu \frac{\partial v}{\partial y}-\beta T-p,
\end{aligned}
$$




$$
\begin{aligned}
& \sigma_{z z}=\lambda\left(\frac{\partial u}{\partial x}+\frac{\partial v}{\partial y}\right)-\beta T-p \\
& \sigma_{x y}=\mu\left(\frac{\partial u}{\partial y}+\frac{\partial v}{\partial x}\right)-\frac{p}{2}\left(\frac{\partial v}{\partial x}-\frac{\partial u}{\partial y}\right) .
\end{aligned}
$$

The basic governing equations of linear magnetic thermoelastic materials under influence of the initial stress become

$$
\begin{gathered}
\left(\mu-\frac{p}{2}\right) \nabla^{2} u+\left(\lambda+\mu+\frac{p}{2}\right) \frac{\partial e}{\partial x}-\beta \frac{\partial T}{\partial x} \\
+\mu_{0} \mathrm{H}_{0}^{2} \frac{\partial e}{\partial x}-\mu_{0}^{2} \mathrm{H}_{0}^{2} \varepsilon_{0} \frac{\partial^{2} u}{\partial t^{2}}=\rho \frac{\partial^{2} u}{\partial t^{2}} \\
\left(\mu-\frac{p}{2}\right) \nabla^{2} v+\left(\lambda+\mu+\frac{p}{2}\right) \frac{\partial e}{\partial y}-\beta \frac{\partial T}{\partial y} \\
+\mu_{0} \mathrm{H}_{0}^{2} \frac{\partial e}{\partial y}-\mu_{0}^{2} \mathrm{H}_{0}^{2} \varepsilon_{0} \frac{\partial^{2} v}{\partial t^{2}}=\rho \frac{\partial^{2} v}{\partial t^{2}} \\
K \nabla^{2} T+k * \frac{\partial}{\partial t} \nabla^{2} T \\
=\rho C_{E} \frac{\partial^{2} T}{\partial t^{2}}+\beta T_{0} \frac{\partial^{2} e}{\partial t^{2}} .
\end{gathered}
$$

Where, $h=-H_{0} e$

For the purpose of numerical evaluation, dimension variables are introduced.

$$
\begin{aligned}
& \left(\mathrm{u}^{\prime}, v^{\prime}\right)=\frac{\omega_{1}^{*}}{c_{1}}(\mathrm{u}, v), T^{\prime}=\frac{T}{T_{0}}, \\
& \left(x^{\prime}, y^{\prime}\right)=\frac{\omega_{1}^{*}}{c_{0}}(x, y), \sigma_{x x}^{\prime}=\frac{\sigma_{x x}}{\mu}, \\
& \sigma_{x y}^{\prime}=\frac{\sigma_{x y}}{\mu}, \sigma_{i j}^{\prime}=\frac{\sigma_{i j}}{\mu_{0}} \sigma_{i j}, \\
& t^{\prime}=\omega_{1}^{*} t, \quad c_{1}^{2}=\frac{\lambda+2 \mu}{\rho}, \\
& \omega_{1}^{*}=\frac{\rho C_{E} c_{1}^{2}}{k}, h^{\prime}=\frac{h}{H_{0}}, \\
& \beta=(3 \lambda+2 \mu) \alpha_{t}, \quad p^{\prime}=\frac{p}{\mu} .
\end{aligned}
$$

Equations (21) - (23), with the help of nondimensional variables (24) may be recast into the dimensionless form after dropping primes for convenience as:

$$
\begin{aligned}
& \nabla^{2} u+E_{1} \frac{\partial e}{\partial x}-E_{2} \frac{\partial T}{\partial x}=E_{3} \frac{\partial^{2} u}{\partial t^{2}}, \\
& \nabla^{2} v+E_{1} \frac{\partial e}{\partial y} E_{2} \frac{\partial T}{\partial y}=E_{3} \frac{\partial^{2} v}{\partial t^{2}}, \\
& \varepsilon_{1} \nabla^{2} T+\varepsilon_{2} \frac{\partial}{\partial t} \nabla^{2} T=\frac{\partial^{2} T}{\partial t^{2}}+\varepsilon_{3} \frac{\partial^{2} e}{\partial t^{2}} .
\end{aligned}
$$

Here,

$$
\begin{aligned}
& E_{1}=\frac{2 \lambda+\mu(2+p)+2 \mu_{0} \mathrm{H}_{0}^{2}}{\mu(2-p)}, \\
& E_{2}=\frac{2 \beta T_{0}}{\mu(2-p)}, \quad E_{3}=\frac{2 \mu_{0} \mathrm{H}_{0}^{2} \varepsilon_{0} c_{1}^{2}+2 \rho c_{1}^{2}}{\mu(2-p)}, \\
& \varepsilon_{1}=\frac{K}{c_{1}^{2} \rho c e}, \quad \varepsilon_{2}=\frac{K \omega_{1}^{*}}{c_{1}^{2} \rho c e}, \quad \varepsilon_{3}=\frac{\beta}{\rho c e} .
\end{aligned}
$$

Where $\varepsilon_{1}, \varepsilon_{2}$, and $\varepsilon_{3}$ are the coupling constants. Using the expression relating displacement components $\mathrm{u}(\mathrm{x}, \mathrm{y}, \mathrm{t})$ and $\mathrm{v}(\mathrm{x}, \mathrm{y}, \mathrm{t})$ to the scalar potential functions

$\psi_{1}(\mathrm{x}, \mathrm{y}, \mathrm{t})$ and $\psi_{2}(\mathrm{x}, \mathrm{y}, \mathrm{t})$ in dimensionless form.

$$
\begin{aligned}
& u=\frac{\partial \psi_{1}}{\partial x}+\frac{\partial \psi_{2}}{\partial y}, \text { and } v=\frac{\partial \psi_{1}}{\partial y}-\frac{\partial \psi_{2}}{\partial x} \\
& e=\nabla^{2} \psi_{1}, \text { and }\left(\frac{\partial u}{\partial y}-\frac{\partial v}{\partial x}\right)=\nabla^{2} \psi_{2} .
\end{aligned}
$$

By substituting from Eq. (29) in Eqs. (25)(27), this yields

$$
\begin{aligned}
& \left(1+E_{1}\right) \nabla^{2} \psi_{1}-E_{2} T=E_{3} \frac{\partial^{2}}{\partial t^{2}} \psi_{1}, \\
& {\left[\nabla^{2}-E_{3} \frac{\partial^{2}}{\partial t^{2}}\right] \psi_{2}=0,} \\
& \varepsilon_{1} \nabla^{2} T+\varepsilon_{2} \frac{\partial}{\partial t} \nabla^{2} T=\frac{\partial^{2} \mathrm{~T}}{\partial t^{2}}+\varepsilon_{3} \frac{\partial^{2} e}{\partial t^{2}} \nabla^{2} \psi_{1} .
\end{aligned}
$$

Then the components of stress tensor will be

$$
\begin{aligned}
& \sigma_{x x}=E_{4}\left(\frac{\partial u}{\partial x}+\frac{\partial v}{\partial y}\right)+2 \frac{\partial u}{\partial x}-E_{2} T-p, \\
& \sigma_{y y}=E_{4}\left(\frac{\partial u}{\partial x}+\frac{\partial v}{\partial y}\right)+2 \frac{\partial v}{\partial y}-E_{2} T-p,
\end{aligned}
$$

(C) 2020 The Author(s). This open access article is distributed under a CC BY-NC 4.0 license. 
$\sigma_{z z}=E_{4}\left(\frac{\partial u}{\partial x}+\frac{\partial v}{\partial y}\right)-E_{2} T-p$,

$\sigma_{x y}=\left(\frac{\partial u}{\partial y}+\frac{\partial v}{\partial x}\right)-\frac{p}{2}\left(\frac{\partial v}{\partial x}-\frac{\partial u}{\partial y}\right)$.

Where $\quad E 4=\frac{\lambda}{\mu}$,

\section{NORMAL MODE ANALYSIS}

The applied methodology to the system was the normal mode analysis to obtain the exact expressions for the used physical variables. The solution of the considered physical variables can be decomposed in terms of the normal mode as the following form

$\left[u, v, T, \psi_{1}, \psi_{2}, \sigma_{i j}\right](x, y, t)=$

$\left[u^{*}, v^{*}, T^{*}, \psi_{1}^{*}, \psi_{2}{ }^{*}, \sigma_{i j}^{*}\right](y) \exp [i(\omega t+a x)]$,

Where $\left[u^{*}, v^{*}, T^{*}, \psi_{1}{ }^{*}, \psi_{2}{ }^{*}, \sigma_{i j}^{*}\right](y)$ are the amplitudes of the function, $\omega$ is the complex time constant, $i=\sqrt{-1}$ and $a$ is the wave number in $x$-direction.

Using Eq. (37) into Eqs. (30)-(32), yields the following,

$$
\begin{aligned}
& \left(\mathrm{D}^{2}-F_{2}\right) \psi_{1}{ }^{*}-F_{3} T^{*}=0, \\
& \left(\mathrm{D}^{2}-F_{4}\right) \psi_{2}{ }^{*}=0, \\
& F_{5}\left(\mathrm{D}^{2}-a^{2}\right) \psi_{1}{ }^{*}+\left(\mathrm{D}^{2}-F_{6}\right) T^{*}=0 .
\end{aligned}
$$

Where,

$$
\begin{aligned}
& \mathrm{D}=\frac{\mathrm{d}}{\mathrm{dy}}, \quad F_{1}=1+E_{1}, \quad F_{2}=\frac{F_{1} a^{2}-E_{3} \omega^{2}}{F_{1}}, \\
& F_{3}=\frac{E_{2}}{F_{1}}, F_{4}=a^{2}-E_{3} \omega^{2}, F_{5}=\frac{\varepsilon_{3} \omega^{2}}{\varepsilon_{1}+\varepsilon_{2} \omega},
\end{aligned}
$$

Eliminating $\psi_{1}^{*}(y)$ and $T^{*}(y)$ between Eqs. (38) - (40), yields the following fourth order ordinary differential equations for $\psi_{1}^{*}(y)$ and $T^{*}(y)$ :

$$
\left[\mathrm{D}^{4}-A \mathrm{D}^{2}+B\right]\left\{\psi_{1}^{*}(y), T^{*}(y)\right\}=0 \text {. }
$$

Equation (41) can be factored as

$$
\left(\mathrm{D}^{2}-k_{1}^{2}\right)\left(\mathrm{D}^{2}-k_{2}^{2}\right)\left\{\psi_{1}^{*}(y), T^{*}(y)\right\}=0 \text {. }
$$

Where $k_{n}^{2}(n=1,2)$ are the roots of the characteristic equation of Eq. (41),

$$
\begin{aligned}
& A=F_{2}+F_{6}+-F_{3} F_{5}, \\
& B=F_{2} F_{6}-F_{3} F_{5} a^{2}, \quad m^{2}=F_{4}=a^{2}-E_{3} \omega^{2} .
\end{aligned}
$$

The solution of Eqs. (41) and (39) have the form

$$
\begin{aligned}
& \psi_{1}^{*}(y)=\sum_{n=1}^{2} G_{n} e^{-k_{n} y}, \\
& T^{*}(y)=\sum_{n=1}^{2} L_{l n} G_{n} e^{-k_{n} y}, \\
& \psi_{2}^{*}=G_{3} e^{-m y} .
\end{aligned}
$$

Where $G_{n}(n=1,2,3)$ are some parameters and $L_{1 n}=\frac{k_{n}^{2}-F_{2}}{F_{3}}$.

Substituting from Eqs. (43)-(45) and (37) in Eqs. (28), (33)-(35) respectively, the displacement and stress components take the form

$$
\begin{aligned}
u^{*} & =\left(\sum_{n=1}^{2} i a G_{n} e^{-k_{n} y}-m G_{3} e^{-m y}\right) e^{(\omega t+i a x)}, \\
v^{*} & =\left(\sum_{n=1}^{2}-k_{n} G_{n} e^{-k_{n} y}-i a G_{3} e^{-m y}\right) e^{(\omega t+i a x)}, \\
\sigma_{x x}^{*} & =\left(\sum_{n=1}^{2} L_{2 n} G_{n} e^{-k_{n} y}-b_{1} G_{3} e^{-m y}\right) e^{i(\omega t+a x)}-p, \\
\sigma_{y y}^{*} & =\left(\sum_{n=1}^{2} L_{3 n} G_{n} e^{-k_{n} y}+b_{1} G_{3} e^{-m y}\right) e^{(\omega t+i a x)}-p, \\
\sigma_{x y}^{*} & =\left(\sum_{n=1}^{2} L_{4 n} G_{n} e^{-k_{n} y}+b_{2} G_{3} e^{-m y}\right) e^{(\omega t+i a x)},
\end{aligned}
$$

Where,

$$
\begin{gathered}
L_{2 \mathrm{n}}=\left(k_{n}^{2}-a^{2}\right) E_{4}+2 k_{n}^{2}-E_{2} L_{1 \mathrm{n}}, \\
L_{3 n}=L_{2 \mathrm{n}}=\left(k_{n}^{2}-a^{2}\right) E_{4}-2 a^{2}-E_{2} L_{1 \mathrm{n}}, \\
L_{4 n}=2 i a k_{n}, \quad b_{1}=2 \mathrm{ia} \mathrm{m}, \\
b_{2}=\left(a^{2}+m^{2}\right)-\frac{p}{2}\left(m^{2}-a^{2}\right) .
\end{gathered}
$$

\section{THE BOUNDARY CONDITIONS}

In this section, the boundary conditions at $y=0$, needs to be considered, in order to determine the constants $G_{\mathrm{n}} \quad(\mathrm{n}=1,2,3)$ :

(1) The mechanical boundary conditions

$$
\sigma_{y y}=-P_{l} e^{(\omega t+\mathrm{i} a x)}, \quad \sigma_{x y}=0,
$$


(2) The thermal boundary condition that the surface of the half-space is subjected to

$T=P_{2} e^{(\omega t+\mathrm{i} a x)}$.

Where $P_{1}$ is the magnitude of the applied force on the half-space and $P_{2}$ is the applied constant temperature to the boundary.

Using the expressions of the variables into the above boundary conditions (51), (52) produces,

$\sum_{n=1}^{2} L_{3 \mathrm{n}} G_{\mathrm{n}}+b_{1} G_{3}=-p_{1}$,

$\sum_{n=1}^{2} L_{4 \mathrm{n}} G_{\mathrm{n}}+b_{1} G_{3}=0$

$\sum_{\mathrm{n}=1}^{3} L_{1 \mathrm{n}} G_{\mathrm{n}}=P_{2}$.

Invoking boundary conditions (53)-(55) at the surface $y=0$ of the plate, yields a system of three equations. After applying the inverse of the matrix method, one can get the values of the three constants $G_{\mathrm{n}}(\mathrm{n}=1,2,3)$.

$$
\left(\begin{array}{l}
G_{1} \\
G_{2} \\
G_{3}
\end{array}\right)=\left(\begin{array}{lll}
L_{31} & L_{32} & b_{1} \\
L_{41} & L_{42} & b_{2} \\
L_{11} & L_{12} & 0
\end{array}\right)^{-1}=\left(\begin{array}{c}
-P_{1} \\
0 \\
P_{2}
\end{array}\right) .
$$

Hence, obtaining the expressions for the displacements, the temperature distribution, and the other physical quantities of the plate surface.

$$
\begin{aligned}
& \lambda=2.17 \times 10^{10} \mathrm{~N} / \mathrm{m}^{2}, \\
& \mu=3.278 \times 10^{10} \mathrm{~N} / \mathrm{m}^{2} \\
& K=1.7 \times 10^{2} \mathrm{~W} / \mathrm{m} \mathrm{deg}, \\
& \alpha_{t}=1.78 \times 10^{-5} \mathrm{~N} / \mathrm{m}^{2}, \\
& \rho=1.74 \times 10^{3} \mathrm{~kg} / \mathrm{m}^{3}, \\
& C_{E}=1.04 \times 10^{3} \mathrm{~J} / \mathrm{kg} \mathrm{deg}, \quad T_{0}=298 \mathrm{~K}, \\
& \beta=2.68 \times 10^{6} \mathrm{~N} / \mathrm{m}^{2} \mathrm{deg}, \quad \omega_{1}^{*}=3.58 \times 10^{11} / \mathrm{s} .
\end{aligned}
$$

The Magnetic field parameters were

$$
\begin{aligned}
& H_{0}=10^{8}, \quad \mu_{0}=4 \pi \times 10^{-7} \mathrm{H} / \mathrm{M}, \\
& \varepsilon_{0}=8.85418717 \times 10^{-12} \mathrm{~F} / \mathrm{M} .
\end{aligned}
$$

The comparisons were carried out for $x=0.5, \quad t=0.3, \quad \omega=\zeta_{0}+i \zeta_{1}, \quad \zeta_{0}=-0.7$, $\xi_{1}=0.1, \quad p_{1}=-0.1, \quad \mathrm{p}_{2}=0.2, \quad a=0.5$, $0 \leq y \leq 25$.

The comparisons have established for two cases

(i) With and without magnetic field

$$
\left[\left(H_{0}=10^{8}, 0\right), \quad p=1, \quad t=0.3\right] \text {. }
$$

(ii) With and without initial stress

$$
\left[(p=1,0), \quad H_{0}=10^{8}, \quad t=0.3\right] .
$$

The above numerical technique, was used for the distribution of the real parts of the displacement components $u$ and $v$, the temperature distribution $T$, the stress components $\sigma_{x x}, \sigma_{y y}$ and $\sigma_{x y}$ with the distance for (G-N) theory of both types II and III with and without the magnetic field $\left(H_{0}=0,10^{8}\right)$ during $p=1$, and $t=0.3$ in figures (1-6).

Figures (7-12) clarify the distribution of the real parts of the displacement components $u$ and $v$, the temperature $T$, the stress components with the distance $y$ for (G-N) theory of both types II and III with and without the initial stress $(p=1,0)$ during $H_{0}=10^{8}$, and $t=0.3$. Figures (1-12) are graphically represented changes in the behavior of the physical quantities against distance $y$ in 2D.

Fig. 1 depicts that the distribution of the vertical displacement $u$, in the context of both types II and III, always begins from positive values for $H_{0}=10^{8}, 0$ and begins from negative values for $H_{0}=10^{8}$ of type III. It was observed that the displacement $u$ increases with the increase of the magnetic field for $y>0$. The distributions of $u$ is directly proportional to the magnetic field. 


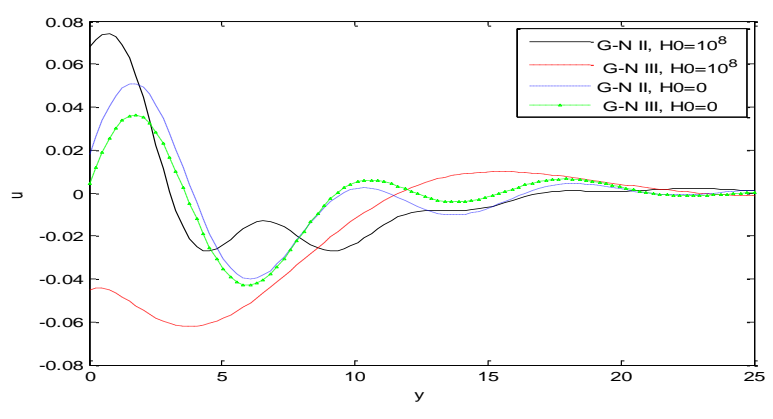

Fig.(1) Horizontal displacement distribution $u$ in the absence and presence of the magnetic field.

Fig. 2 depicts the displacement distribution $v$, in the context of both types II and III for $H_{0}=10^{8}, 0$ it was observed that the distributions of $v$ decrease with the increase of the magnetic field for $y>0$. The distributions of $v$ are inversely proportional to the magnetic field.

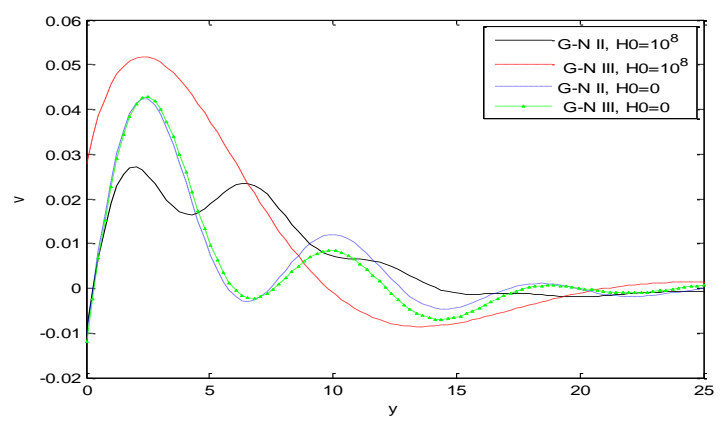

Fig.(2) Vertical displacement distribution $v$ in the absence and presence of the magnetic field.

Fig. 3 explains that the distribution of temperature $T$ begins from a positive value (which is the same point) in case of $H_{0}=0,10^{8}$, in the context of both types II and III of (G-N), and takes the form of a wave until it develops to zero.

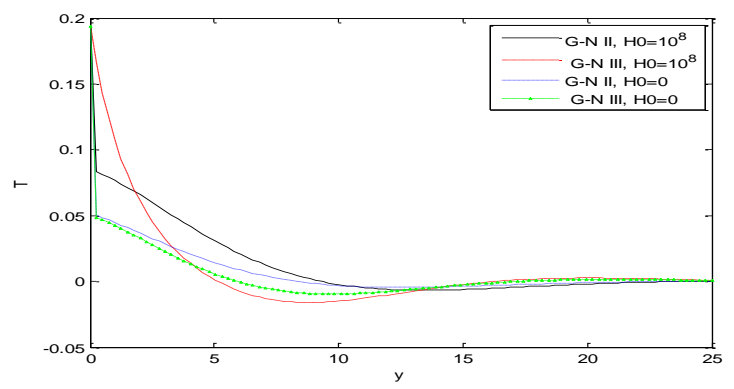

Fig. (3) Thermodynamic temperature distribution $T$ in the absence and presence of magnetic field.

(C) 2020 The Author(s). This open access article is distributed under a CC BY-NC 4.0 license.

ISSN: online 2617-2186 print 2617-2178
Fig. 4 determines the distribution of the stress component $\sigma_{x x}$ in the case of $H_{0}=10^{8}$, and $H_{0}=0$, in the context of both types II and III. It was noted that the distribution of $\sigma_{x x}$ decreases with the increase of the magnetic field value for $y>0$.

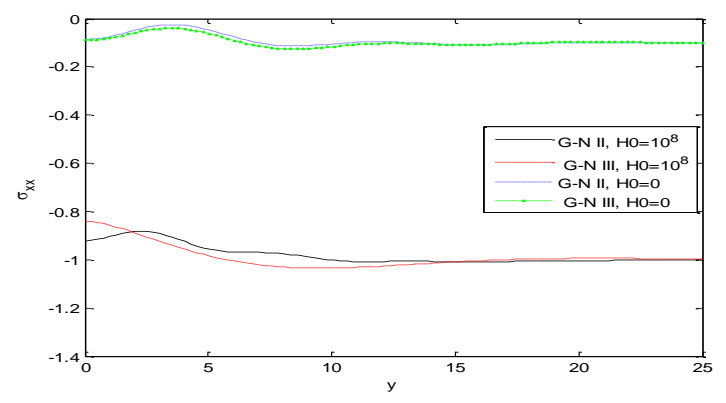

Fig. (4) Distribution of stress component $\sigma_{x x}$ in the absence and presence of the magnetic field.

Fig. 5 shows the distribution of the stress component $\sigma_{y y}$ in the case of $H_{0}=10^{8}, 0$, in the context of both types II and III. It was observed that the distribution of $\sigma_{y y}$ decreases with the increase of the magnetic field value for $y>0$.

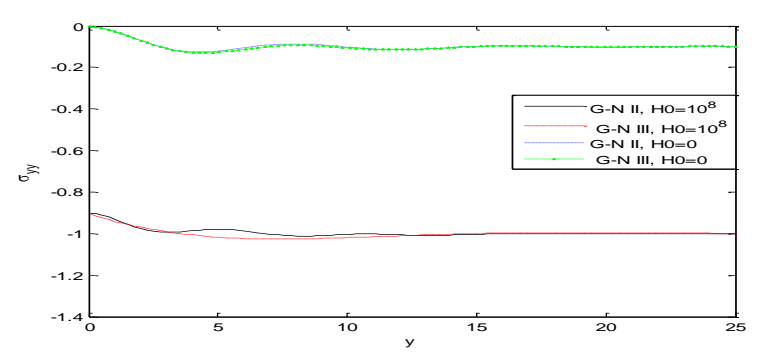

Fig. (5) Distribution of stress component $\sigma_{x y}$ in the absence and presence of the magnetic field.

Fig. 6 explains the distribution of stress component $\sigma_{x y}$ which begins from zero in the case of $H_{0}=10^{8}, H_{0}=0$, in the context of both types II and III. It was observed that the magnetic field has an effect on $\sigma_{x y}$, while the distribution of $\sigma_{x y}$ increases with the increase of the magnetic field value for $y>0$. 


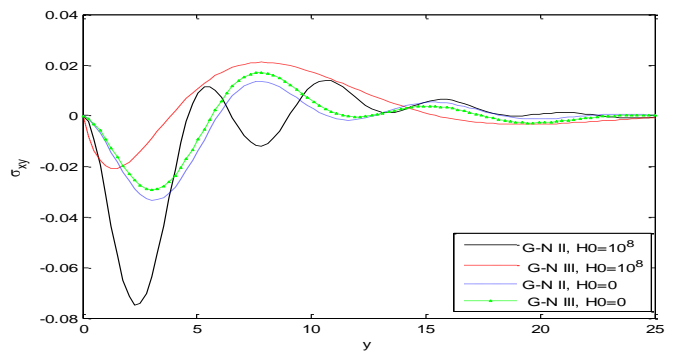

Fig. (6) Distribution of stress component $\sigma_{x y}$ in the absence and presence of magnetic field.

Figs. 7 and 8 show the distribution of displacement components $u$ and $v$ in the case of $p=1$ and $p=0$, in the context of both types II and III. It was noted that the distributions of $u$ and $v$ respectively increase with the increase of the initial stress for $y>0$. The distributions of $u$ and $v$ are directly proportional to the initial stress.

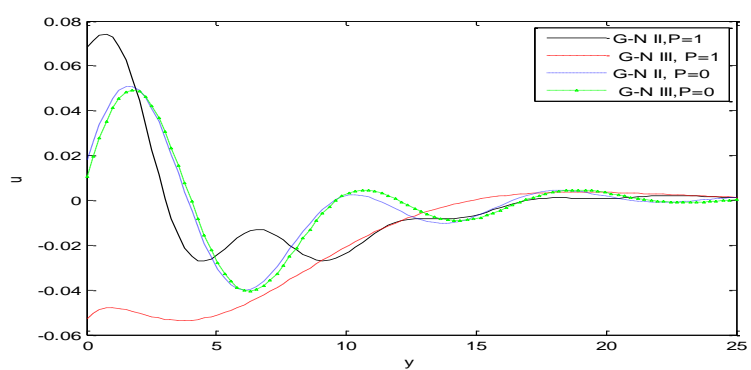

Fig. (7) Distribution of the displacement component $u$ with and without initial stress.

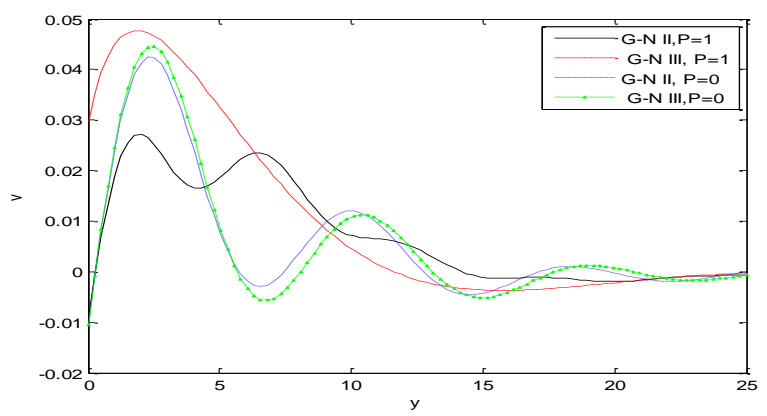

Fig. (8) Distribution of the displacement component $v$ with and without initial stress.

Figs. 9 explains the distribution of temperature $\mathrm{T}$ which begins from zero in the case of $p=1$ and $p=0$, in the context of both types II and III. It was noticed that $\mathrm{T}$ decreases with the increase of the initial stress for $y>0$.

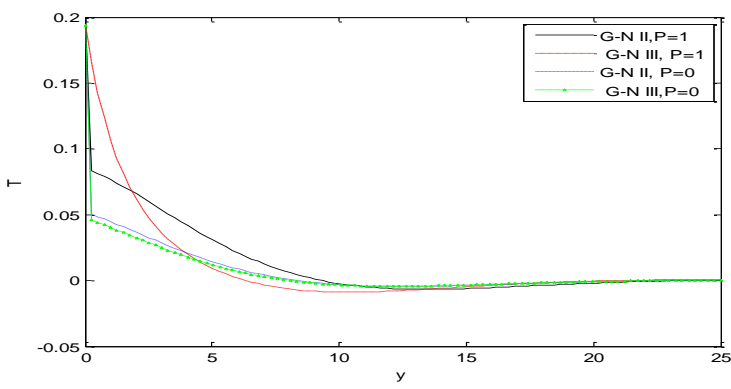

Fig. (9) Temperature distribution $T$ with and without initial stress.

Figs. 10 and 11 depict the behavior of $\sigma_{x x}$ and $\sigma_{y y}$ in the context of both types II and III which always begin from positive values for $p=1,0$, and begin from negative for $p=1$ in type II. It was observed that stress components $\sigma_{x x}$ and $\sigma_{y y}$ increase with the increase of the initial stress for $y>0$.

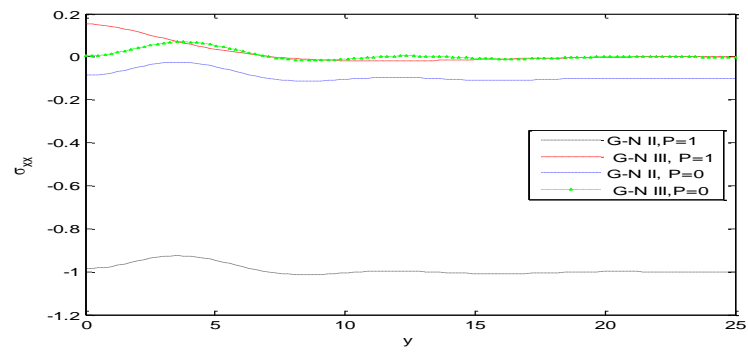

Fig. (10) Distribution of the stress component $\sigma_{x x}$ with and without initial stress.

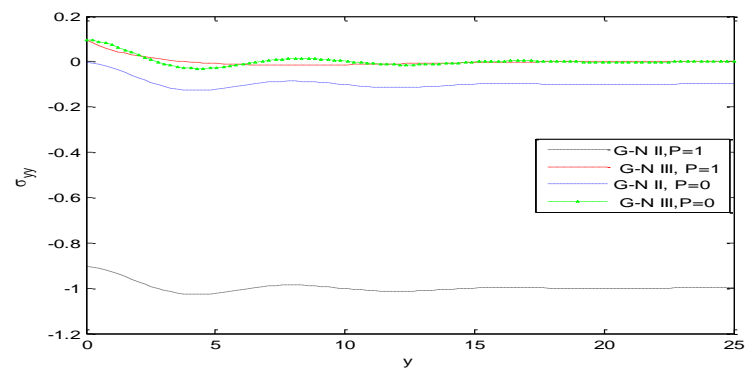

Fig. (11) Distribution of the stress component $\sigma_{y y}$ with and without initial stress.

Figs. 12 demonstrates that the distribution of the stress component $\sigma_{x y}$, in the context of both types II and III begins from zero and satisfies the boundary conditions at $p=1$, and $p=0$. In the context of both types II and III. It was noted that the stress component of $\sigma_{x y}$, 
increases with the increase of the initial stress values for $y>0$.

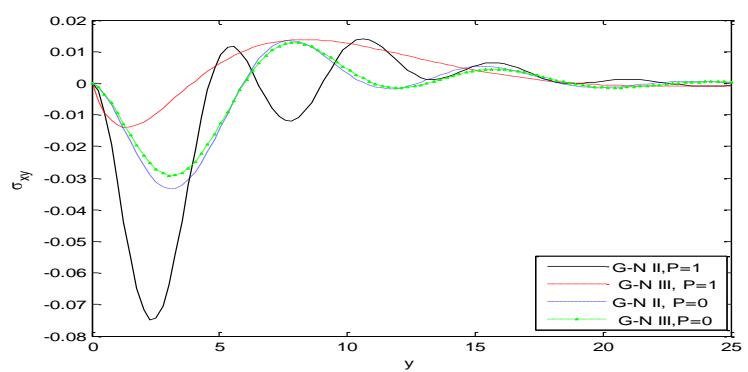

Fig. (12) Distribution of the stress component $\sigma_{x y}$ with and without initial stress.

$3 \mathrm{D}$ curves are representing $y=0$ the complete relation between the physical variables and both of the components of the distance as shown in Figures (13-18) in the presence of the magnetic field $H_{0}=10^{8}$ and the initial stress proprieties $p=1$, at $t=0.3$ in the context of (G-N) theory of type III. These figures are very important in studying the dependence of these physical quantities on the vertical component of the distance. The obtained curves are highly dependent on the vertical distance from the origin, and all the physical quantities are moving in the wave propagation.

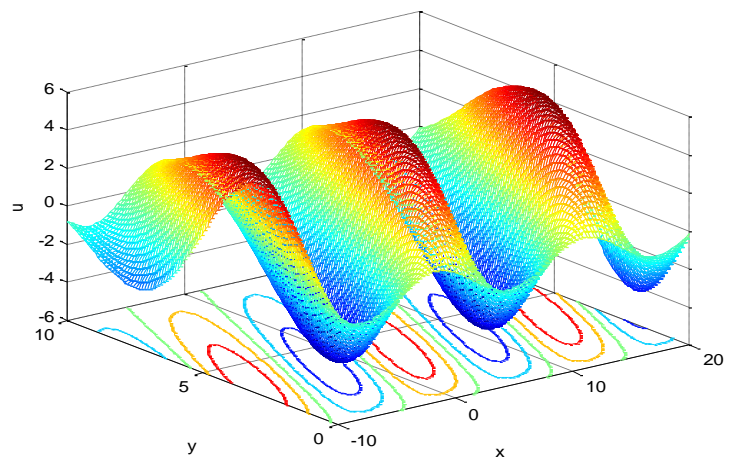

Fig. 13 (3D) Horizontal component of displacement $u$ against both components of distance based on G-N type III at $H_{0}=10^{8}$ and $P=1$.

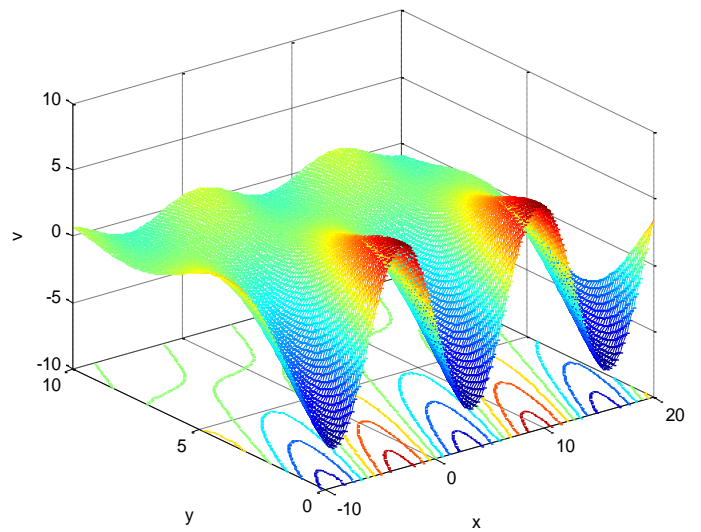

Fig. 14 (3D) Vertical component of displacement $v$ against both components of distance based on G-N type III at $H_{0}=10^{8}$ and $P=1$.

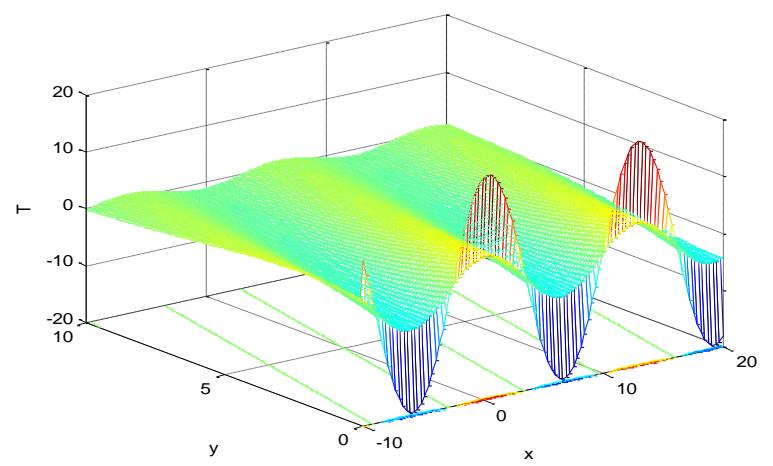

Fig. 15 (3D) Thermodynamic temperature distribution $T$ against both components of distance based on G-N type III at $H_{0}=10^{8}$ and $P=1$.

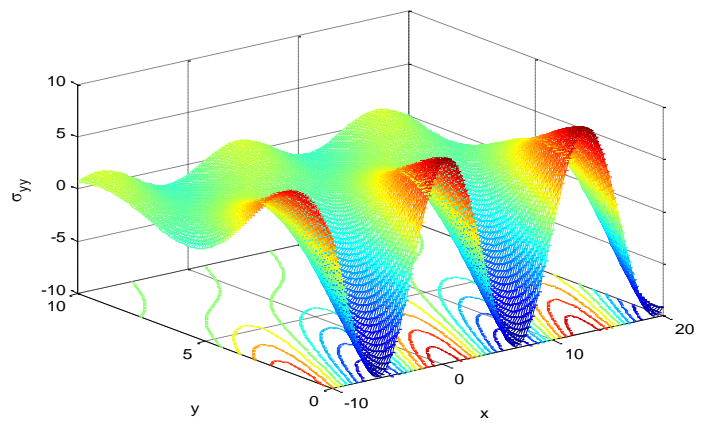

Fig. 16 (3D) Distribution of the stress component $\sigma_{y y}$ against both components of distance based on G-N type III at $H_{0}=10^{8}$ and $P=1$. 


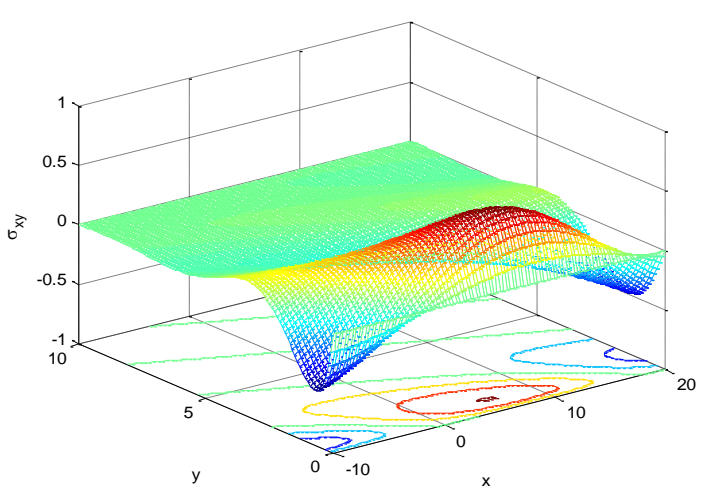

Fig. 17 (3D) Distribution of the stress component $\sigma_{x y}$ against both components of distance based on G-N type III at $H_{0}=10^{8}$ and $P=1$.

\section{CONCLUDING REMARKS}

By comparing the figures obtained under the (G-N) theory in the context of both types II and III, important phenomena are observed: the values of all physical quantities converge to zero with increasing distance $y$, all functions are continuous, and all physical quantities satisfy the boundary conditions. Also, analysis of the components of displacement, stresses, the temperature distribution due to the initial stress, and the magnetic field for thermoelastic solid with magnetic field under the initial stress is an interesting problem of mechanics. Normal mode analysis technique has been used, which applies to a wide range of problems in thermoelasticity. The value of all physical quantities converges to zero, with an increase in distance and all functions are continuous y. It was observed that the magnetic field and initial stress have a significant role in all considered physical quantities, as the amplitudes of these quantities vary (increasing or decreasing) with the increase of the initial stress and magnetic field.

\section{REFERENCES}

Abd-Alla, A., El-Naggar, A., \& Fahmy, M.
(2003). Magneto-thermoelastic problem in non-homogeneous isotropic cylinder. Heat and Mass transfer, 39(7), 625629.

Abd-Elaziz, E. M., Marin, M., \& Othman, M. I. (2019). On the effect of Thomson and initial stress in a thermo-porous elastic solid under GN electromagnetic theory. Symmetry, 11(3), 413.

Abo-Dahab, S., Abd-Alla, A., \& Alqarni, A. (2017). A two-dimensional problem with rotation and magnetic field in the context of four thermoelastic theories. Results in physics, 7, 2742-2751.

Ailawalia, P., Khurana, G., \& Kumar, S. (2009). Effect of rotation in a generalized thermoelastic medium with two temperature under the influence of gravity. International Journal of Applied Mathematics and Mechanics, 5(5), 99-116.

Ailawalia, P., \& Narah, N. S. (2009). Effect of rotation in a generalized thermoelastic medium with hydrostatic initial stress subjected to ramp-type heating and loading. International Journal of Thermophysics, 30(6), 2078-2097.

Atwa, S. Y. (2014). Generalized magnetothermoelasticity with two temperature and initial stress under Green-Naghdi theory. Applied Mathematical Modelling, 38(21-22), 5217-5230.

Bargmann, S., \& Steinmann, P. (2006). Theoretical and computational aspects of non-classical thermoelasticity. Computer Methods in Applied Mechanics and Engineering, 196(1-3), 516-527.

Chandrasekharaiah, D. (1998). Hyperbolic thermoelasticity: a review of recent literature. 
Choudhuri, S. R., \& Debnath, L. (1985). Magneto-thermo-elastic plane waves in generalized thermoelasticity. $J$. Elasticity, 15(1), 59-68.

Green, A., \& Naghdi, P. (1993). Thermoelasticity without energy dissipation. Journal of elasticity, 31(3), 189-208.

Green, A. E., \& Lindsay, K. A. (1972). Thermoelasticity. Journal of elasticity, 2(1), 1-7.

Hetnarski, R. B., \& Ignaczak, J. (1999). Generalized thermoelasticity. Journal of Thermal Stresses, 22(4-5), 451-476.

Lord, H. W., \& Shulman, Y. (1967). A generalized dynamical theory of thermoelasticity. Journal of the Mechanics and Physics of Solids, 15(5), 299-309.

Montanaro, A. (1999). On singular surfaces in isotropic linear thermoelasticity with initial stress. The Journal of the Acoustical Society of America, 106(3), 1586-1588.

Othman, M., \& Edeeb, E. (2016). Effect of initial stress on generalized magnetothermoelasticity medium with voids: a comparison of different theories. Int $J$ Eng Math Comput Sci, 4(5), 15-26.

Othman, M., \& Song, Y. (2006). The effect of rotation on the reflection of magnetothermoelastic waves under thermoelasticity without energy dissipation. Acta Mechanica, 184(1), 189-204.

Othman, M. A., \& Atwa, S. Y. (2011). The effect of magnetic field on 2-D problem of generalized thermoelasticity with energy dissipation. International
Journal of Industrial Mathematics, 3(3), 213-226.

Othman, M. I., \& Atwa, S. Y. (2012). Thermoelastic plane waves for an elastic solid half-space under hydrostatic initial stress of type III. Meccanica, 47(6), 1337-1347.

Othman, M. I., Atwa, S. Y., Jahangir, A., \& Khan, A. (2013a). Effect of magnetic field and rotation on generalized thermo-microstretch. Elastic solid with mode-I crack under the Green Naghdi theory. Computational Mathematics and Modeling, 24(4), 566-591.

Othman, M. I., Atwa, S. Y., Jahangir, A., \& Khan, A. (2013b). Generalized magneto - thermo - microstretch elastic solid under gravitational effect with energy dissipation. Multidiscipline Modeling in Materials and Structures.

Othman, M. I., \& Kumar, R. (2009). Reflection of magneto-thermoelasticity waves with temperature dependent properties in generalized thermoelasticity. International Communications in Heat and Mass Transfer, 36(5), 513-520.

Othman, M. I., \& Song, Y. (2007). Reflection of plane waves from an elastic solid half-space under hydrostatic initial stress without energy dissipation. International Journal of Solids and Structures, 44(17), 5651-5664.

Paria, G. (1966). Magneto-elasticity and magneto-thermo-elasticity. In Advances in Applied Mechanics (Vol. 10, pp. 73112). Elsevier.

Sherief, H. H., \& Helmy, K. A. (2002). A twodimensional problem for a half-space in magneto-thermoelasticity with thermal 
relaxation. International Journal of Engineering Science, 40(5), 587-604.

Singh, B. (2008). Effect of hydrostatic initial stresses on waves in a thermoelastic solid half-space. Applied Mathematics and Computation, 198(2), 494-505.

Singh, B., Kumar, A., \& Singh, J. (2006). Reflection of generalized thermoelastic waves from a solid half-space under hydrostatic initial stress. Applied Mathematics and Computation, 177(1), 170-177.

Tzou, D. Y. (1995). A unified field approach for heat conduction from macro-to micro-scales. 


\section{تحليل الوضع الطبيعي للوسط المغناطيسي الحراري المرن المعمم مع إجهاد أولي في ظل نظرية (جرين وناخدي)}

الزائرة رمضان محمد الذيب

قسم الرياضيات، جامعة الزاوبية، كلية التربية الزاوبية، لبييا

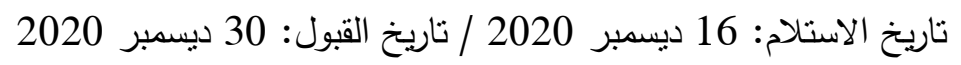
https://doi.org/10.54172/mjsc.v35i4.330 :Doi

المستخلص: تم استخدام طريقة تحليل الوضـع الطبيعـي في دراسـة تأثير كل من الإجهاد الأساسـي الهيدروستانيكي، والمجـال

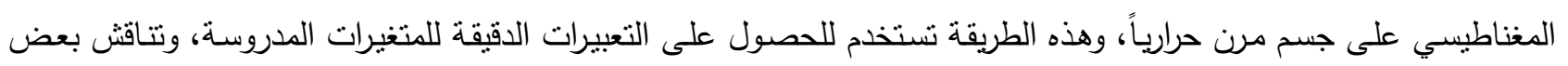
الحالات الخاصة أيضا في سياق المشكلة ـ حيث نم استعراض معادلات المرونة الحرارية المعممة تحت تأثير الإجهاد الأساسي الهيدروستانيكي، والمجال المغناطيسي باستخدام نظرية (جرين وناخدي) من النوعين الثاني والثالث (النوع الثاني مع عدم نتنت

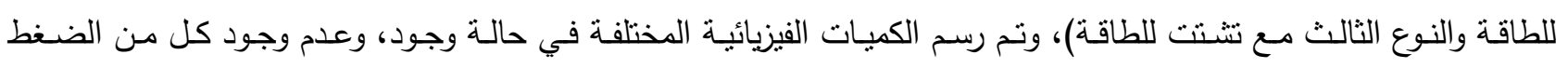

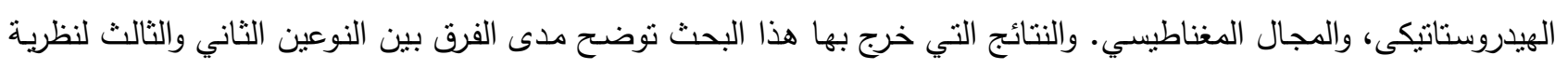

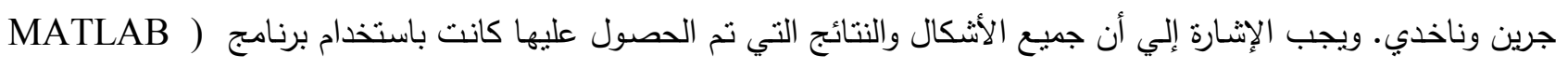
(R2013a

الكلمات المفتاحية: المرونة الحرارية المعمدة. المجال المغناطيسي، الإجهاد الأساسي العيدروستانيكي، طريقة تحليل الوضع الطبيعي، نظرية (جرين وناخدي). 